\title{
Molecular taxonomy of Dunaliella (Chlorophyceae), with a special focus on $D$. salina: ITS2 sequences revisited with an extensive geographical sampling
}

Patrícia Assunção ${ }^{1 *}$, Ruth Jaén-Molina ${ }^{2}$, Juli Caujapé-Castells ${ }^{2}$, Adelina de la Jara ${ }^{1}$, Laura Carmona ${ }^{1}$, Karen Freijanes ${ }^{1}$ and Héctor Mendoza ${ }^{1 *}$

\begin{abstract}
We used an ITS2 primary and secondary structure and Compensatory Base Changes (CBCs) analyses on new French and Spanish Dunallela salina strains to investigate their phylogenetic position and taxonomic status within the genus Dunaliella. Our analyses show a great diversity within D. salina (with only some clades not statistically supported) and reveal considerable genetic diversity and structure within Dunaliella, although the CBC analysis did not bolster the existence of different biological groups within this taxon. The ITS2 sequences of the new Spanish and French D. salina strains were very similar except for two of them: ITC5105 "Janubio" from Spain and ITC5119 from France. Although the Spanish one had a unique ITS2 sequence profile and the phylogenetic tree indicates that this strain can represent a new species, this hypothesis was not confirmed by $\mathrm{CBCs}$, and clarification of its taxonomic status requires further investigation with new data. Overall, the use of CBCs to define species boundaries within Dunaliella was not conclusive in some cases, and the ITS2 region does not contain a geographical signal overall.
\end{abstract}

Keywords: Canary Islands, Compensatory Base Changes, Dunaliella salina, Internal Transcribed Spacer, Saltworks, Taxonomy

\section{Background}

The Internal Transcribed Spacer 2 (ITS2) of the nuclear rDNA cistron is one of the most frequently used regions for phylogenetic analysis in algae [1-3]. Although its application in deep taxonomic levels was initially limited to comparisons of genera within the same family owing to uncertainties in alignment at higher taxonomic levels, the analysis of its secondary structure has provided key solutions to this problem [4]. Thus, the use of an ITS2 secondary structure improves sequence alignments, resulting in a higher robustness and accuracy of phylogenetic reconstructions [5] and providing help to distinguish species [6]. Furthermore, an automatic approach

\footnotetext{
*Correspondence: pclemente@itccanarias.org; hmendoza@itccanarias.org 'Departamento de Biotecnología. División de Investigación y Desarrollo Tecnológico. Instituto Tecnológico de Canarias (ITC). Pozo Izquierdo, 35119 Sta. Lucía, Canary Islands, Spain

Full list of author information is available at the end of the article
}

to analysis is possible [7], as a pipeline consisting of the ITS2 Database (annotation/structure prediction), 4SALE (alignment), ProfDistS (inferring phylogenies) and the CBCAnalyzer (distinguishing species) have recently become available (http://its2.bioapps.biozentrum.uniwuerzburg.de/?about).

In Dunaliella (Chlorophyceae), the use of ITS2 secondary structure for phylogenetic analysis has a long tradition [8-13]. The genus Dunaliella comprises twenty-eight recognized species separated in two subgenera, Pascheria (which contains the freshwater species), and Dunaliella (grouping the marine species); the latter is further subdivided into four sections: Tertiolecta, Dunaliella, Viridis and Peirceinae [11]. The species ascribed to these four sections occur in a wide range of marine habitats such as oceans, brine lakes, salt marshes, salt lagoons and salt water dishes near the sea [14], being Dunaliella salina Teodoresco (section

\section{Biomed Central}


Dunaliella) the most studied one. Dunaliella salina, is the most halotolerant eukaryotic photosynthetic organism known to date $[14,15]$ since it shows a remarkable degree of adaptation to a variety of salt concentrations and it accumulates large amounts of carotenes under extremely stressful conditions such as high salinity, low nitrogen levels, and high solar radiation [14]. Nowadays, it is the best commercial source of natural $\beta$-carotene $[14,16]$, and it also stands out as a source of glycerol [17].

One of the aspects of $D$. salina that have most intrigued researchers is the enormous variability within strains regarding its geographic, physiological, and morphological characteristics [18-24]. Recent phylogenetic analyses of ITS1+ITS2 combined with the analysis of the ITS2 secondary structure of $D$. salina strains have also revealed a high intraspecific variation [8-11,25].

The high genetic diversity detected in D. salina, its morphological plasticity, and the restricted geographical sampling used in all scientific publications to date have hindered taxonomic elucidation in this taxon. Our objectives are (1) to use the ITS2 sequences and secondary structure analysis in a thorough geographic and taxonomic representation of the genus Dunaliella and particularly $D$. salina (including new Spanish and French strains) to improve understanding of the complex phylogenetic structure in this taxon, (2) to study the relationship of the new strains with the Dunaliella sequences available at the ITS2 Database and/or at GenBank [26]; (3) to investigate if the Compensatory Base Changes (CBCs) analyses could elucidate the species concept in Dunaliella, and reveal potentially new species; and (4) to test if D. salina ITC5105 "Janubio" could be considered a new species.

\section{Results}

The ITS2 primary and secondary structure phylogenetic analysis of all the Dunaliella sequences available in the ITS2 Database plus the new sequences revealed great heterogeneity, although some of the clades were not statistically supported (Figure 1). No phylogenetic relationship is supported between the two Dunaliella freshwater species, since $D$. lateralis was positioned outside the Dunaliella subgenus, while D. acidophila was positioned within this subgenus.

The strains positioned in the tertiolecta-clade represent species that belong to different traditionally accepted sections: section Tertiolecta (D. tertiolecta, D. primolecta, D. quartolecta, D. polymorpha), section Viridis (D. bioculata, D. minuta), section Dunaliella (D. salina, D. parva), and section Peircei (D. percei). The majority of these strains had an exact ITS2 sequence (Figure 1). Most D. viridis strains sampled were positioned in a single clade; however, some strains
(UTEX200, CCAP19/3) were positioned in a different clade together with $D$. pseudosalina CONC010 (pseudosalina-clade), but without statistical support.

The majority of the $D$. salina strains were distributed in two different clades (salina-clade-I and salina-clade II), positioned between the tertiolecta sub-clades, whereas two D. salina strains (CCAP19/30, CCAP19/18) were positioned together in a different clade. Only two of the new ITS2 D. salina sequences, ITC5119 ("salinaclade-I") and D. salina ITC5105 "Janubio" (salina-clade II), had a unique ITS2 sequence profile (Figure 1). The $\mathrm{CBC}$ analysis of the ITS2 secondary structure showed that there is at least one CBC between "Janubio" and the other $D$. salina strains analyzed (see Additional file 1), except D. salina CCAP19/30 and CCAP19/18; however these two strains were phylogenetically distant to Janubio.

The taxonomic identification of the Dunaliella sp. strains (below the $D$. acidophila strain in Figure 1) was not possible because they did not match with any known Dunaliella species. Despite the phylogenetic tree indicates that this group may indeed represent a new species; the analysis of the CBC did not confirm these results (Additional file 1 ).

We observed a lack of CBCs in some strains when we compared species in different clades: viridis $v s$ salina, viridis $v s$ tertiolecta, salinas $v s$ tertiolecta, salina $v s$ salina, etc (Additional File 1). Also, the CBC analysis of the different species within the subgenus Dunaliella for the confirmation of the species boundaries was not conclusive in some cases (Additional file 1).

The phylogenetic results of the ITS2 sequences in this study, and previous confirmed identification of some Dunaliella strains (see Table 1), allow us to suggest the re-identification of some strains (Table 1).

Finally, the ITS2 data was not informative regarding the geographic origin of the $D$. salina strains.

\section{Discusion}

The ITS2 Database allows automatic large scale simultaneous analyses of both ITS2 sequences and their secondary structures. Potential pitfalls are in structures obtained by different algorithms; however the main difficulty of performing a phylogenetic analysis of the genus Dunaliella is the misinformation available at Culture Collections and GenBank regarding the identification of strains and sequences. The tracking of the true identification of each strain was only possible after consulting several publications where the authors concluded that they were misidentified and suggested their re-identification $[9,10,27]$. In this study, we have also suggested the re-identification of some strains based on our ITS2 data. To overcome all these unnecessary problems (and given that it is unlikely that all the Dunaliella strains could be 


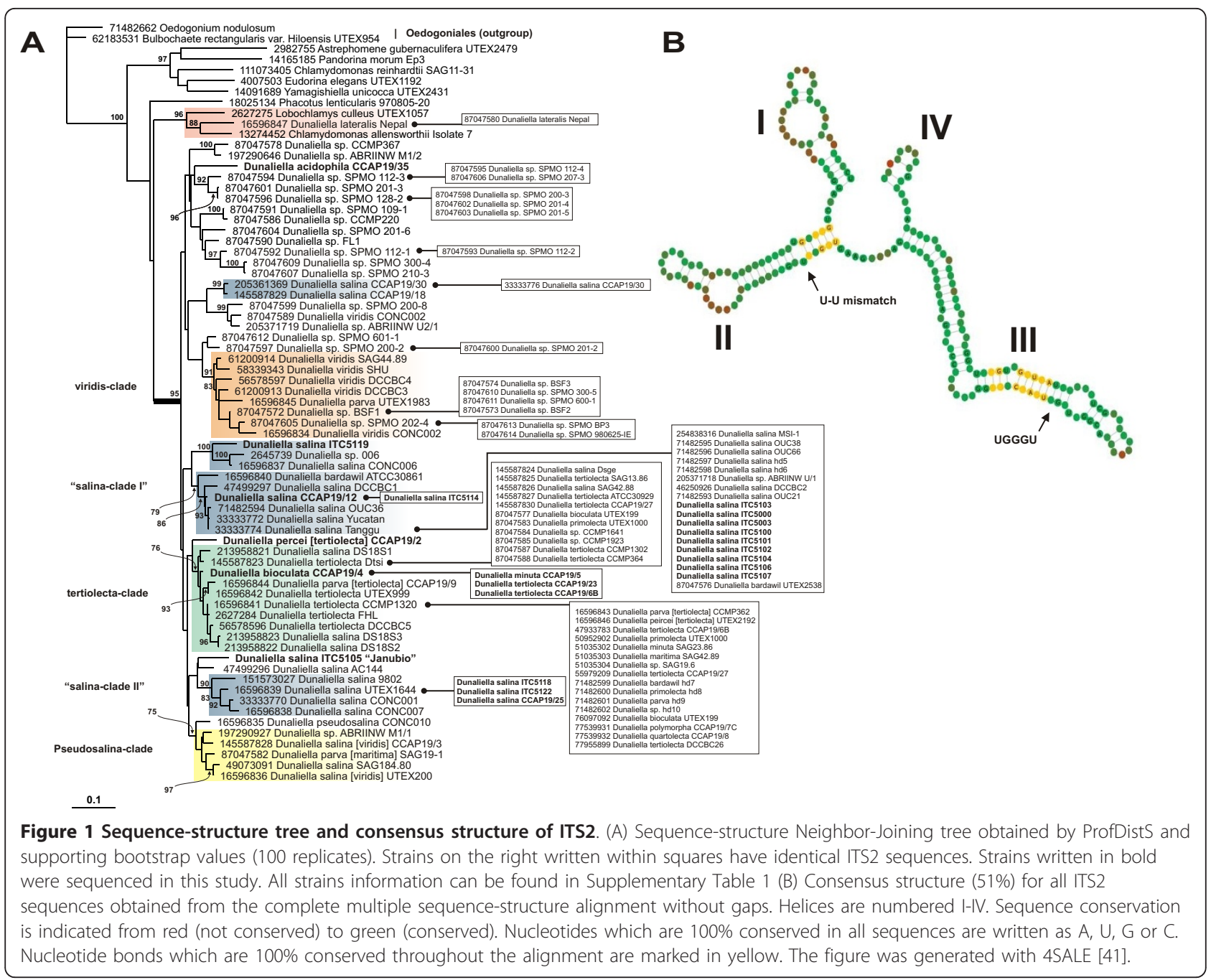

openly available), we suggest to establish a "type strain" for each Dunaliella taxon (including subspecies, forms or varieties). These basic data should be easily obtained from any official culture collection, thereby greatly facilitating comparison with new field isolates and avoiding misleading information and/or false conclusions.

Our ITS2 phylogenetic analysis of Dunaliella reveals several major groups, and positions the freshwater $D$. lateralis clearly outside Dunaliella, confirming that it no longer should be considered a member of this genus [10]. Nevertheless, the other freshwater species analyzed in this study (D. acidophila, CCAP19/35), maintained its position within the subgenus Dunaliella, and was not phylogenetically related to $D$. lateralis, as recently proposed [28]. Furthermore, the observation that different species belonging to several Sections (Tertiolecta, Viridis, Dunaliella and Peircei) share the exact same ITS2 sequence, make us believe that they correspond to a single species. These data agree with other authors $[10,11,27,29]$, who suggested that the number of
Dunaliella species may be much lower than it has been claimed till now. The possibility that the ITS2 gene is not able to discriminate between these species is highly unlikely; therefore, our observations support the suggestion that the morphological and physiological criteria available to discriminate Dunaliella species are either not very reliable [11], or are difficult to interpret..

In an attempt to clarify the species concept within Dunaliella, we searched for compensatory base changes (CBCs). Several case studies have revealed that the detection of a $C B C$ in the ITS2 secondary structure between two organisms is correlated with sexual incompatibility $[6,30,31]$, and these changes have been proposed as markers for distinguishing species $[6,7,30,31]$. In summary, these investigations conclude that while a $\mathrm{CBC}$ in a pair of sequences is positively correlated with species distinctness at a confidence level of $93 \%$, the lack of a CBC in the ITS2 secondary structure does not necessarily indicate that two organisms belong to the same species [6]. The overall analysis of the CBC was 
Table 1 Taxonomic classification, Culture Collection, Geographic Origin and GenBank acession numbers of the strains included in this study.

\begin{tabular}{|c|c|c|c|c|c|c|}
\hline Former classification & $\begin{array}{l}\text { New suggested classification } \\
\text { [Reference] and comments }\end{array}$ & $\begin{array}{l}\text { Culture } \\
\text { Collection }\end{array}$ & Geographic Origin & $\begin{array}{l}\text { Isolator } \\
\text { (Date) }\end{array}$ & $\begin{array}{l}\text { GenBank } \\
\text { acession } \\
\text { number }\end{array}$ & $\begin{array}{l}\text { GenBank } \\
\text { identifier }\end{array}$ \\
\hline \multicolumn{7}{|c|}{ Subgenus Pascheria (Freshwater species) } \\
\hline \multirow{2}{*}{$\begin{array}{l}\text { Dunaliella lateralis } \\
\text { Pascher \&Jahoda }\end{array}$} & & & Nepal & & AF313445 & 16596847 \\
\hline & & & Nepal & & DQ377089 & 87047580 \\
\hline $\begin{array}{l}\text { Dunaliella acidophila } \\
\text { (Kalina) Massyuk }\end{array}$ & & CCAP19/35 & $\begin{array}{l}\text { Freshwater; acidic } \\
\text { sulphurous pool, Pisciarelli, } \\
\text { Naples, Italy }\end{array}$ & $\begin{array}{l}\text { Albertano } \\
\text { (1981) }\end{array}$ & HM060646 & \\
\hline \multicolumn{7}{|l|}{ Subgenus Dunaliella } \\
\hline \multicolumn{7}{|c|}{ Section Tertiolecta (Marine species. Optimum salinity $<6 \% \mathrm{NaCl}$ ) } \\
\hline \multirow[t]{14}{*}{$\begin{array}{l}\text { Dunaliella tertiolecta } \\
\text { Butcher }\end{array}$} & & CCAP19/6B & Brackish; Oslo Fjord, Norway & $\begin{array}{l}\text { Foyn (1928 } \\
\text { or earlier) }\end{array}$ & HM243579* & \\
\hline & & CCAP19/6B & Brackish; Oslo Fjord, Norway & $\begin{array}{l}\text { Foyn }(1928 \\
\text { or earlier) }\end{array}$ & AY572957 & 47933783 \\
\hline & & CCAP19/27 & & & EF473748 & 145587830 \\
\hline & & CCAP19/27 & & & AY654300 & 55979209 \\
\hline & & Dtsi & Italy: Venezia & & EF473730 & 145587823 \\
\hline & & UTEX999 & Norway: Oslofjord & & AF313435 & 16596842 \\
\hline & & CCMP1320 & Salt flat. USA? & & AF313433 & 16596841 \\
\hline & & CCMP1302 & Salt flat. USA? & & DQ377096 & 87047587 \\
\hline & & CCMP364 & Salt flat. USA? & & DQ377097 & 87047588 \\
\hline & & $\mathrm{FHL}$ & & & DSU66956 & 2627284 \\
\hline & & DCCBC5 & & & AY686684 & 56578596 \\
\hline & & SAG13.86 & Norway: Oslofjord & & EF473738 & 145587825 \\
\hline & & ATCC30929 & United Kingdom: Plymouth & & EF473742 & 145587827 \\
\hline & & DCCBC26 & & & DQ224338 & 77955899 \\
\hline $\begin{array}{l}\text { Dunaliella quartolecta } \\
\text { Butcher }\end{array}$ & Dunaliella tertiolecta [this study] & CCAP19/8 & $\begin{array}{l}\text { Marine; Southampton, } \\
\text { Hampshire, England }\end{array}$ & $\begin{array}{l}\text { Butcher } \\
\text { (1953) }\end{array}$ & DQ157054 & 77539932 \\
\hline \multirow[t]{3}{*}{$\begin{array}{l}\text { Dunaliella primolecta } \\
\text { Butcher }\end{array}$} & Dunaliella tertiolecta [this study] & UTEX1000 & $\begin{array}{l}\text { English Channel, Plymouth, } \\
\text { Devon, England }\end{array}$ & Gross (1936) & AY582942 & 50952902 \\
\hline & & UTEX1000 & $\begin{array}{l}\text { English Channel, Plymouth, } \\
\text { Devon, England }\end{array}$ & Gross (1936) & DQ377092 & 87047583 \\
\hline & Dunaliella tertiolecta [this study] & hd8 & China? & & DQ116745 & 71482600 \\
\hline $\begin{array}{l}\text { Dunaliella polymorpha } \\
\text { Butcher }\end{array}$ & Dunaliella tertiolecta [this study] & CCAP19/7C & $\begin{array}{l}\text { Brackish; River Crouch, Essex, } \\
\text { England }\end{array}$ & $\begin{array}{l}\text { Butcher } \\
\text { (1954) }\end{array}$ & DQ157053 & 77539931 \\
\hline $\begin{array}{l}\text { Dunaliella marítima } \\
\text { Butcher }\end{array}$ & Dunaliella tertiolecta [this study] & SAG42.89 & & & AY582086 & 51035303 \\
\hline \multicolumn{7}{|c|}{ Section Dunaliella (Halophilic species. Optimum salinity $>6 \% \mathrm{NaCl}$. Accumulates carotenes) } \\
\hline \multirow[t]{11}{*}{$\begin{array}{l}\text { Dunaliella salina } \\
\text { Teodoresco }\end{array}$} & & CCAP19/18 & $\begin{array}{l}\text { Hypersaline; Hypersaline } \\
\text { brines, Hutt Lagoon, } \\
\text { Western Australia }\end{array}$ & $\begin{array}{l}\text { Kaethner } \\
\text { (1982) }\end{array}$ & AF546098 & 33333776 \\
\hline & & CCAP19/18 & $\begin{array}{l}\text { Hypersaline; Hypersaline } \\
\text { brines, Hutt Lagoon, } \\
\text { Western Australia }\end{array}$ & $\begin{array}{l}\text { Kaethner } \\
\text { (1982) }\end{array}$ & EF473746 & 145587829 \\
\hline & & $\underline{\text { CCAP19/25 }}$ & & & HM140783 & \\
\hline & & $\underline{\text { UTEX1644 }}$ & $\begin{array}{l}\text { Point Colorado Salinas; La } \\
\text { Paz, Baja California, Mexico }\end{array}$ & $\begin{array}{l}\text { Loeblich } \\
\text { (1967) }\end{array}$ & AF313429 & 16596839 \\
\hline & & CONCO06 & Salar de Atacama, Chile & $(1990)$ & AF313425 & 16596837 \\
\hline & & CONC001 & Laguna La Rinconada, Chile & & AF546092 & 33333770 \\
\hline & & CONCO07 & Salar de Atacama, Chile & $(1990)$ & AF313427 & 16596838 \\
\hline & & DCCBC1 & Lake Tyrell, Victoria, Australia & Polle & AY549442 & 47499297 \\
\hline & & $\mathrm{DCCBC2}$ & South Korea & & AY512973 & 46250926 \\
\hline & & hd6 & Israel & & DQ116743 & 71482598 \\
\hline & & & Yucatan, Mexico & & AF546094 & 33333772 \\
\hline
\end{tabular}


Table 1 Taxonomic classification, Culture Collection, Geographic Origin and GenBank acession numbers of the strains included in this study. (Continued)

\begin{tabular}{|c|c|c|c|c|c|}
\hline & & $\overline{\text { Tanggu, China }}$ & & AF546096 & 33333774 \\
\hline & AC144 & Tunisia, North Africa & & AY549441 & 47499296 \\
\hline Dunaliella viridis [this study] & 184.80 & & & AY577766 & 49073091 \\
\hline & $\begin{array}{l}\text { OUC66 } \\
\text { "hd4" }\end{array}$ & China & (2005) ? & DQ116741 & 71482596 \\
\hline & $\begin{array}{l}\text { OUC38 } \\
\text { "hd3" }\end{array}$ & China & (2005) ? & DQ116740 & 71482595 \\
\hline & $\begin{array}{l}\text { OUC36 } \\
\text { "hd2" }\end{array}$ & China & (2005) ? & DQ116739 & 71482594 \\
\hline & $\begin{array}{l}\text { OUC21 } \\
\text { "hd1" }\end{array}$ & China & (2005) ? & DQ116738 & 71482593 \\
\hline & 9802 & China? & (2007) ? & EF695405 & 151573027 \\
\hline & "hd5" & Inner-Mongolia & (2005) & DQ116742 & 71482597 \\
\hline Dunaliella tertiolecta [this study] & DS18S1 & Mexico? & & FJ360756 & 213958821 \\
\hline Dunaliella tertiolecta [this study] & DS18S2 & Mexico? & & FJ360757 & 213958822 \\
\hline Dunaliella tertiolecta [this study] & DS18S3 & Mexico? & & FJ360758 & 213958823 \\
\hline Dunaliella tertiolecta [this study] & Dsge & Belgium: Gent & & EF473732 & 145587824 \\
\hline Dunaliella viridis $[9,10,27]$ & CCAP $19 / 3$ & $\begin{array}{l}\text { Brackish; dirty salt lake, } \\
\text { Soviet Union }\end{array}$ & Mainx & EF473744 & 145587828 \\
\hline Dunaliella viridis $[9,10,27]$ & UTEX200 & $\begin{array}{l}\text { Brackish; dirty salt lake, } \\
\text { Soviet Union }\end{array}$ & Mainx & AF313423 & 16596836 \\
\hline & MSI-1 & & & GQ337903 & 254838316 \\
\hline & ITC5100 & Vargas, Gran Canaria, Spain & $\begin{array}{l}\text { de la Jara \& } \\
\text { Mendoza } \\
\text { (2005) }\end{array}$ & HM035353* & \\
\hline & ITC5101 & Punta, Gran Canaria, Spain & $\begin{array}{l}\text { de la Jara \& } \\
\text { Mendoza } \\
\text { (2005) }\end{array}$ & HM035354* & \\
\hline & ITC5102 & Tenefé, Gran Canaria, Spain & $\begin{array}{l}\text { de la Jara \& } \\
\text { Mendoza } \\
\text { (2005) }\end{array}$ & HM035355* & \\
\hline & ITC5103 & Rio, Lanzarote, Spain & $\begin{array}{l}\text { de la Jara \& } \\
\text { Mendoza } \\
\text { (2005) }\end{array}$ & HM035356* & \\
\hline & ITC5104 & Guatiza, Lanzarote, Spain & $\begin{array}{l}\text { de la Jara \& } \\
\text { Mendoza } \\
\text { (2005) }\end{array}$ & HM035357* & \\
\hline & ITC5105 & Janubio, Lanzarote, Spain & $\begin{array}{l}\text { de la Jara \& } \\
\text { Mendoza } \\
\text { (2005) }\end{array}$ & HM035346* & \\
\hline & ITC5106 & $\begin{array}{l}\text { Carmen (Majo), } \\
\text { Fuerteventura, Spain }\end{array}$ & $\begin{array}{l}\text { Mendoza \& } \\
\text { Trujillano } \\
\text { (2003) }\end{array}$ & HM035358* & \\
\hline & ITC5107 & Añana, Álava, Spain & $\begin{array}{l}\text { de la Jara \& } \\
\text { Mendoza } \\
\text { (2005) }\end{array}$ & HM035359* & \\
\hline & ITC5118 & île de Ré (01), France & $\begin{array}{l}\text { Carmona \& } \\
\text { Mendoza } \\
\text { (2006) }\end{array}$ & HM035348* & \\
\hline & ITC5122 & île de Ré (05), France & $\begin{array}{l}\text { Carmona \& } \\
\text { Mendoza } \\
(2006)\end{array}$ & HM035347* & \\
\hline & ITC5114 & La Tapa, Cádiz, Spain & $\begin{array}{l}\text { de la Jara \& } \\
\text { Mendoza } \\
\text { (2007) }\end{array}$ & HM035350* & \\
\hline & ITC5119 & île de Ré (02), France & $\begin{array}{l}\text { Carmona \& } \\
\text { Mendoza } \\
\text { (2006) }\end{array}$ & HM035349* & \\
\hline
\end{tabular}


Table 1 Taxonomic classification, Culture Collection, Geographic Origin and GenBank acession numbers of the strains included in this study. (Continued)

\begin{tabular}{|c|c|c|c|c|c|c|}
\hline & Aliquot of Dunaliella salina BCA421 & ITC5003 & Tenefe, Gran Canaria, Spain & $\begin{array}{l}\text { Mendoza } \\
\text { (1992) }\end{array}$ & HM035352* & \\
\hline \multirow[t]{6}{*}{$\begin{array}{l}\text { Dunaliella bardawil } \\
\text { nomen nudum Ben- } \\
\text { Amotz \& Avron }\end{array}$} & $\begin{array}{l}\text { Dunaliella salina CCAP 19/30 [27], } \\
\text { obtained from Dr. Joao Varela (Faro, } \\
\text { Portugal) }\end{array}$ & ITC5000 & $\begin{array}{l}\text { Marine; salt pond, near } \\
\text { Bardawil lagoon, North Sinai, } \\
\text { Israel }\end{array}$ & $\begin{array}{l}\text { Ben-Amotz } \\
\text { \& Avron } \\
\text { (1976). }\end{array}$ & HM035351* & \\
\hline & $\begin{array}{l}\text { Dunaliella salina [27]. Reinstated } \\
\text { from SAG on April } 1996\end{array}$ & CCAP19/30 & $\begin{array}{l}\text { Marine; salt pond near } \\
\text { Bardawil lagoon, North Sinai, } \\
\text { Israel }\end{array}$ & $\begin{array}{l}\text { Ben-Amotz } \\
\& \text { Avron } \\
\text { (1976). }\end{array}$ & EU932917 & 205361369 \\
\hline & Dunaliella salina [27] & ATCC30861 & $\begin{array}{l}\text { Marine; salt pond near } \\
\text { Bardawil lagoon, North Sinai, } \\
\text { Israel }\end{array}$ & $\begin{array}{l}\text { Ben-Amotz } \\
\& \text { Avron } \\
(1976)\end{array}$ & AF313431 & 16596840 \\
\hline & Dunaliella salina [27] & $\underline{\text { UTEX2538 }}$ & $\begin{array}{l}\text { Marine; salt pond near } \\
\text { Bardawil lagoon, North Sinai, } \\
\text { Israel }\end{array}$ & $\begin{array}{l}\text { Ben-Amotz } \\
\& \text { Avron } \\
(1978)\end{array}$ & DQ377085 & 87047576 \\
\hline & $\begin{array}{l}\text { Dunaliella salina [27], Dunaliella } \\
\text { tertiolecta [this study] }\end{array}$ & $\underline{\mathrm{SAG} 42.88}$ & $\begin{array}{l}\text { Marine; salt pond, near. } \\
\text { Bardawil lagoon, North Sinai, } \\
\text { Israel }\end{array}$ & $\begin{array}{l}\text { Ben-Amotz } \\
\& \text { Avron } \\
(1976)\end{array}$ & EF473741 & \\
\hline & Dunaliella tertiolecta [this study] & hd7 & China? & & DQ116744 & 71482599 \\
\hline \multirow[t]{5}{*}{ Dunaliella parva Lerche } & Dunaliella viridis [9-11] & UTEX1983 & Dead Sea & (1973) & AF313441 & 16596845 \\
\hline & $\begin{array}{l}\text { Dunaliella tertiolecta }[10,11] \text { Dunaliella } \\
\text { quartolecta [27] }\end{array}$ & CCAP19/9 & $\begin{array}{l}\text { Brackish; salt marsh, Northey } \\
\text { Island, Essex, England }\end{array}$ & $\begin{array}{l}\text { Butcher } \\
\text { (1956) }\end{array}$ & AF313439 & 16596844 \\
\hline & Dunaliella tertiolecta $[10,11]$ & CCMP362 & & Gold & AF313437 & 16596843 \\
\hline & $\begin{array}{l}\text { Dunaliella marítima [27], Dunaliella } \\
\text { viridis [this study] }\end{array}$ & SAG19-1 & Marine: Lacul Sarat, Romania & $\begin{array}{l}\text { Lerche } \\
\text { (Before } \\
\text { 1938) }\end{array}$ & DQ377091 & 87047582 \\
\hline & Dunaliella tertiolecta [this study] & hd9 & China? & & DQ116746 & 71482601 \\
\hline $\begin{array}{l}\text { Dunaliella pseudosalina } \\
\text { Massyuk \& Radchenko }\end{array}$ & & CONC010 & Salar de Atacama, Chile & & AF313421 & 16596835 \\
\hline \multicolumn{7}{|c|}{ Section Viridis (Halophilic species. Optimum salinity > 6\% NaCl. Cells always green. Do not accumulate carotenes. Cells radially symmetrical) } \\
\hline \multirow[t]{2}{*}{ Dunaliella minuta Lerche } & Dunaliella tertiolecta [this study] & CCAP19/5 & $\begin{array}{l}\text { Marine; sand and sea water, } \\
\text { Roscoff, France }\end{array}$ & $\begin{array}{l}\text { Jowett } \\
\text { (1967) }\end{array}$ & HM035345* & \\
\hline & Dunaliella tertiolecta [this study] & SAG23.86 & & & AY582085 & 51035302 \\
\hline \multirow[t]{3}{*}{$\begin{array}{l}\text { Dunaliella bioculata } \\
\text { Butcher }\end{array}$} & Dunaliella tertiolecta [this study] & CCAP19/4 & $\begin{array}{l}\text { Brackish; salt lake, Soviet } \\
\text { Union }\end{array}$ & Mainx & HM035344* & \\
\hline & Dunaliella tertiolecta [this study] & $\underline{\text { UTEX199 }}$ & $\begin{array}{l}\text { Brackish; salt lake, Soviet } \\
\text { Union }\end{array}$ & Mainx & DQ157433 & 76097092 \\
\hline & & UTEX199 & $\begin{array}{l}\text { Brackish; salt lake, Soviet } \\
\text { Union }\end{array}$ & Mainx & DQ377086 & 87047577 \\
\hline \multirow[t]{6}{*}{ Dunaliella viridis Massyuk } & & CONCOO2 & Salar de Atacama, Chile & (1990) & AF313419 & 16596834 \\
\hline & & CONCOO2 & Salar de Atacama, Chile & (1990) & DQ377098 & 87047589 \\
\hline & & SAG44.89 & & & 87047600 & 61200914 \\
\hline & & $\mathrm{SHU}$ & China? & & AY878700 & 58339343 \\
\hline & & DCCBC4 & Great Salt Lake, Utah, USA & & AY686685 & 56578597 \\
\hline & & $\mathrm{DCCBC} 3$ & Great Salt Lake, Utah, USA & & AY828227 & 61200913 \\
\hline
\end{tabular}

Section Peirceinae ((Halophilic species. Optimum salinity > 6\% NaCl. Cells always green. Do not accumulate carotenes. Cells bilaterally symmetrical)

Dunaliella percei Nicolai Dunaliella tertiolecta $[10,11]$

\& Baas-Becking

Dunaliella tertiolecta $[10,11]$

Unknown Dunaliella Species

Dunaliella sp. Dunaliella tertiolecta [27]

Dunaliella salina [27]

Dunaliella tertiolecta [this study]
CCAP19/2

UTEX2192 Brackish; California, USA

CCAP19/23 Marine;

CCAP19/12 Brackish; North Sinai, Israel

CCMP367 Salt flat

CCMP220 Salt flat

CCMP1923 Salt flat

CCMP1641 Salt flat
Nicolai HM035343*

(1931)

Nicolai AF313443

(1931)

Pennick

HM035341*

Ginzburg

(1976)

HM035342*

DQ377087

DQ377095

87047578

DQ377094

87047586

DQ377093 
Table 1 Taxonomic classification, Culture Collection, Geographic Origin and GenBank acession numbers of the strains included in this study. (Continued)

\begin{tabular}{|c|c|c|c|c|c|}
\hline \multirow[t]{2}{*}{ Dunaliella tertiolecta [this study] } & \multicolumn{3}{|l|}{ SAG19.6 } & \multirow{2}{*}{$\begin{array}{l}\text { AY582086 } \\
\text { DQ377099 }\end{array}$} & \multirow{2}{*}{$\begin{array}{l}51035303 \\
87047590\end{array}$} \\
\hline & FL1 & Salt flat & & & \\
\hline Dunaliella viridis [this study] & BSF1 & $\begin{array}{l}\text { USA: Utah, Bonneville Salt } \\
\text { Flats }\end{array}$ & $\begin{array}{l}\text { William } \\
\text { Henley }\end{array}$ & DQ377081 & 87047572 \\
\hline Dunaliella viridis [this study] & BSF2 & $\begin{array}{l}\text { USA: Utah, Bonneville Salt } \\
\text { Flats }\end{array}$ & $\begin{array}{l}\text { William } \\
\text { Henley }\end{array}$ & DQ377082 & 87047573 \\
\hline Dunaliella viridis [this study] & BSF3 & $\begin{array}{l}\text { USA: Utah, Bonneville Salt } \\
\text { Flats }\end{array}$ & $\begin{array}{l}\text { William } \\
\text { Henley }\end{array}$ & DQ377083 & 87047574 \\
\hline Dunaliella salina [this study] & 006 & & $\begin{array}{l}\text { A.W. } \\
\text { Coleman, U. } \\
\text { Brown }\end{array}$ & AF033278 & 2645739 \\
\hline Dunaliella tertiolecta [this study] & hd10 & & & DQ116747 & 71482602 \\
\hline \multirow[t]{2}{*}{ Dunaliella viridis [this study] } & $\begin{array}{l}\text { ABRIINW } \\
\text { M1/1 }\end{array}$ & & & EU927374 & 197290927 \\
\hline & $\begin{array}{l}\text { ABRIINWW } \\
\text { M1/2 }\end{array}$ & Iran? & & EU927373 & 197290646 \\
\hline Dunaliella salina [this study] & $\begin{array}{l}\text { ABRIINW } \\
\text { U1/1 }\end{array}$ & Iran? & & FJ164063 & 205371718 \\
\hline \multirow[t]{14}{*}{ Dunaliella viridis [this study] } & $\begin{array}{l}\text { ABRIINWW } \\
\text { U2/1 }\end{array}$ & Iran? & & FJ164064 & 205371719 \\
\hline & $\begin{array}{l}\text { SPMO112- } \\
3\end{array}$ & $\begin{array}{l}\text { Salt flat, USA: Oklahoma, Salt } \\
\text { Plains National Wildlife } \\
\text { Refuge }\end{array}$ & & DQ377103 & 87047594 \\
\hline & $\begin{array}{l}\text { SPMO201- } \\
3\end{array}$ & $\begin{array}{l}\text { Salt flat, USA: Oklahoma, Salt } \\
\text { Plains National Wildlife } \\
\text { Refuge }\end{array}$ & & DQ377110 & 87047601 \\
\hline & $\begin{array}{l}\text { SPMO128- } \\
2\end{array}$ & $\begin{array}{l}\text { Salt flat, USA: Oklahoma, Salt } \\
\text { Plains National Wildlife } \\
\text { Refuge }\end{array}$ & & DQ377105 & 87047596 \\
\hline & $\begin{array}{l}\text { SPMO109- } \\
1\end{array}$ & $\begin{array}{l}\text { Salt flat, USA: Oklahoma, Salt } \\
\text { Plains National Wildlife } \\
\text { Refuge }\end{array}$ & & DQ377105 & 87047596 \\
\hline & $\begin{array}{l}\text { SPMO112- } \\
4\end{array}$ & $\begin{array}{l}\text { Salt flat, USA: Oklahoma, Salt } \\
\text { Plains National Wildlife } \\
\text { Refuge }\end{array}$ & & DQ377104 & 87047595 \\
\hline & $\begin{array}{l}\text { SPMO207- } \\
3\end{array}$ & $\begin{array}{l}\text { Salt flat, USA: Oklahoma, Salt } \\
\text { Plains National Wildlife } \\
\text { Refuge }\end{array}$ & & DQ377115 & 87047606 \\
\hline & $\begin{array}{l}\text { SPMO200- } \\
3\end{array}$ & $\begin{array}{l}\text { Salt flat, USA: Oklahoma, Salt } \\
\text { Plains National Wildlife } \\
\text { Refuge }\end{array}$ & & DQ377107 & 87047598 \\
\hline & $\begin{array}{l}\text { SPMO201- } \\
4\end{array}$ & $\begin{array}{l}\text { Salt flat, USA: Oklahoma, Salt } \\
\text { Plains National Wildlife } \\
\text { Refuge }\end{array}$ & & DQ377111 & 87047602 \\
\hline & $\begin{array}{l}\text { SPMO201- } \\
5\end{array}$ & $\begin{array}{l}\text { Salt flat, USA: Oklahoma, Salt } \\
\text { Plains National Wildlife } \\
\text { Refuge }\end{array}$ & & DQ377112 & 87047603 \\
\hline & $\begin{array}{l}\text { SPMO201- } \\
6\end{array}$ & $\begin{array}{l}\text { Salt flat, USA: Oklahoma, Salt } \\
\text { Plains National Wildlife } \\
\text { Refuge }\end{array}$ & & DQ377113 & 87047604 \\
\hline & $\begin{array}{l}\text { SPMO112- } \\
1\end{array}$ & $\begin{array}{l}\text { Salt flat, USA: Oklahoma, Salt } \\
\text { Plains National Wildlife } \\
\text { Refuge }\end{array}$ & & DQ377101 & 87047592 \\
\hline & $\begin{array}{l}\text { SPMO112- } \\
2\end{array}$ & $\begin{array}{l}\text { Salt flat, USA: Oklahoma, Salt } \\
\text { Plains National Wildlife } \\
\text { Refuge }\end{array}$ & & DQ377102 & 87047593 \\
\hline & $\begin{array}{l}\text { SPMO300- } \\
4\end{array}$ & $\begin{array}{l}\text { Salt flat, USA: Oklahoma, Salt } \\
\text { Plains National Wildlife } \\
\text { Refuge }\end{array}$ & & DQ377118 & 87047609 \\
\hline
\end{tabular}


Table 1 Taxonomic classification, Culture Collection, Geographic Origin and GenBank acession numbers of the strains included in this study. (Continued)

\begin{tabular}{|c|c|c|c|c|c|}
\hline & $\begin{array}{l}\text { SPMO210- } \\
3\end{array}$ & $\begin{array}{l}\text { Salt flat, USA: Oklahoma, Salt } \\
\text { Plains National Wildlife } \\
\text { Refuge }\end{array}$ & & DQ377116 & 87047607 \\
\hline Dunaliella viridis [this study] & $\begin{array}{l}\text { SPMO200- } \\
8\end{array}$ & $\begin{array}{l}\text { Salt flat, USA: Oklahoma, Salt } \\
\text { Plains National Wildlife } \\
\text { Refuge }\end{array}$ & & DQ377108 & 87047599 \\
\hline Dunaliella viridis [this study] & $\begin{array}{l}\text { SPMO601- } \\
1\end{array}$ & $\begin{array}{l}\text { Salt flat, USA: Oklahoma, Salt } \\
\text { Plains National Wildlife } \\
\text { Refuge }\end{array}$ & & DQ377121 & 87047612 \\
\hline Dunaliella viridis [this study] & $\begin{array}{l}\text { SPMO200- } \\
2\end{array}$ & $\begin{array}{l}\text { Salt flat, USA: Oklahoma, Salt } \\
\text { Plains National Wildlife } \\
\text { Refuge }\end{array}$ & & DQ377106 & 87047597 \\
\hline Dunaliella viridis [this study] & $\begin{array}{l}\text { SPMO201- } \\
2\end{array}$ & $\begin{array}{l}\text { Salt flat, USA: Oklahoma, Salt } \\
\text { Plains National Wildlife } \\
\text { Refuge }\end{array}$ & & DQ377109 & 87047600 \\
\hline Dunaliella viridis [this study] & $\begin{array}{l}\text { SPMO202- } \\
4\end{array}$ & $\begin{array}{l}\text { Salt flat, USA: Oklahoma, Salt } \\
\text { Plains National Wildlife } \\
\text { Refuge }\end{array}$ & & DQ377114 & 87047605 \\
\hline Dunaliella viridis [this study] & $\begin{array}{l}\text { SPMO300- } \\
5\end{array}$ & $\begin{array}{l}\text { Salt flat, USA: Oklahoma, Salt } \\
\text { Plains National Wildlife } \\
\text { Refuge }\end{array}$ & & DQ377119 & 87047610 \\
\hline Dunaliella viridis [this study] & $\begin{array}{l}\text { SPMO600- } \\
1\end{array}$ & $\begin{array}{l}\text { Salt flat, USA: Oklahoma, Salt } \\
\text { Plains National Wildlife } \\
\text { Refuge }\end{array}$ & & DQ377120 & 87047611 \\
\hline Dunaliella viridis [this study] & SPMO BP3 & $\begin{array}{l}\text { Salt flat, USA: Oklahoma, Salt } \\
\text { Plains National Wildlife } \\
\text { Refuge }\end{array}$ & & DQ377122 & 87047613 \\
\hline \multirow[t]{11}{*}{ Dunaliella viridis [this study] } & $\begin{array}{l}\text { SPMO } \\
980625-\mathrm{IE}\end{array}$ & $\begin{array}{l}\text { Salt flat, USA: Oklahoma, Salt } \\
\text { Plains National Wildlife } \\
\text { Refuge }\end{array}$ & & DQ377123 & 87047614 \\
\hline & SAG11-31 & & & AJ749628 & 111073405 \\
\hline & isolate 7 & & & AF326855 & 13274452 \\
\hline & & & & DQ078301 & 71482662 \\
\hline & UTEX954 & $\begin{array}{l}\text { Catawmont, Massachusetts, } \\
\text { USA }\end{array}$ & Cook 1962 & AY962677 & 62183531 \\
\hline & UTEX2479 & & & AGU66932 & 2982755 \\
\hline & EP3 & & & AF378359 & 14165185 \\
\hline & UTEX 1192 & & & AF098173 & 4007503 \\
\hline & UTEX2431 & & & AF375785 & 14091689 \\
\hline & $970805-20$ & & & AY009933 & 18025134 \\
\hline & UTEX1057 & Maxville, Florida, USA & Smith & CCU66946 & 2627275 \\
\hline
\end{tabular}

Other groups

Chlamydomonas reinhardtii

Chlamydomonas

allensworthii

Oedogonium nodulosum

Bulbochaete rectangularis

var. hiloensis

Astrephomene

gubernaculifera

Pandorina morum

Eudorina elegans

Yamagishiella unicocca

Phacotus lenticularis

Lobochlamys culleus

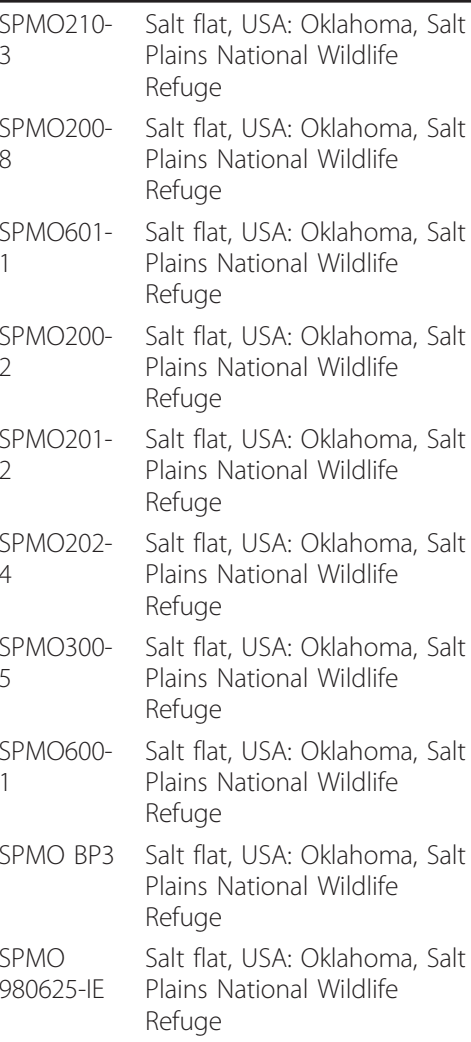


currently known as $D$. salina $[10,11,32]$, indicating the possibility of cryptic speciation [10]. Our ITS2 phylogenetic analysis does confirm the existence of three different groups within $D$. salina; however, the $\mathrm{CBC}$ results did not resolve if these groups may correspond to distinct species, although several strains of each $D$. salina group shared one $\mathrm{CBC}$ with $D$. salina strains in other groups. On the other hand, the high morphological and physiological variability found within the Spanish $D$. salina strains under the same lab conditions [24] was not correlated with the phylogenetic observations of this study. This finding indicates that the phylogenetic closeness found with ITS sequences does not reflect common physiological or morphological attributes. Moreover, our data unequivocally suggest that $D$. salina is not monophyletic, at odds with previous hypotheses $[10,11,32]$.

The other objective of this work was to elucidate if the physiological uniqueness found in "Janubio" [such as its unique fatty acid profile and accumulation of high levels of carotenes under low light flux density conditions; Mendoza et al.: A new strategy for carotenogenesis under conditions of cellular stress in Dunaliella (a Potential New Species), submitted] could be confirmed by the ITS2 data and the CBC analysis. However, although we observed that this strain has a unique ITS2 sequence profile, and had more than one $\mathrm{CBC}$ with the other phylogenetically related $D$. salina, our data do not allow us to conclude that this is a new species, and further studies must be performed to find out if the differences observed are just reflecting a high intra-specific variability. Finally, in agreement with previous studies $[8,11,25]$, our ITS2 data failed to furnish evidence for isolation by distance among $D$. salina strains.

\section{Conclusion}

This work demonstrates that the taxonomy of Dunaliella should be revised. The great diversity observed within the ITS2 sequences of $D$. salina suggests that different biological groups may exist within this taxon; however, this was not confirmed with the $\mathrm{CBC}$ analysis. Likewise, although the Spanish D. salina strain ITC5105 "Janubio" was characterized by a unique ITS2 sequence, the hypothesis that it may be a new species could not be confirmed by the CBCs analysis, requiring further morpho-physiological and genetic investigation. Overall, the use of CBCs to define species boundaries within Dunaliella was not conclusive in some of the cases assessed.

\section{Methods}

\section{Strains, DNA extraction and ITS amplification}

We sequenced the ITS2 region of $13 \mathrm{D}$. salina strains from Spanish and French saltworks, one strain obtained from the Culture Collection of Algae and Protozoa UK
(CCAP), and one D. salina strain that has been maintained in the Instituto Tecnológico de Canarias (ITC henceforth) for several years (purchased from CCAP as Dunaliella salina 19/30). We also sequenced other Dunaliella species (D. minuta CCAP19/5, D. tertiolecta CCAP19/23 and CCAP19/6B, D. bioculata CCAP19/4) [Table 1]. The sequences of the other strains analyzed in this study were obtained from the ITS2 Database (http://its2.bioapps.biozentrum.uni-wuerzburg.de/? about). Detailed information about the strains used in this study can be found in Table 1 .

DNA extraction was performed with a chelex-100 (Biorad, CA, USA) resin-based protocol [33]. For the DNA amplification of the ITS region, primers AB28 and TW81 in Goff et al. (1994) [34] were used. DNA amplification was carried out in a total volume of $25 \mu \mathrm{l}$ with 1X iQ SYBR Green Supermix (Biorad, CA, USA) and 10 pM of each primer in a Smart Cycler thermocyler (Cepheid,CA, USA) as follows: $5 \mathrm{~min}$ at $94^{\circ} \mathrm{C}$; 5 cycles of 1 min at $94^{\circ} \mathrm{C}, 2 \mathrm{~min}$ at $50^{\circ} \mathrm{C}$ and $1 \mathrm{~min}$ at $72^{\circ} \mathrm{C} ; 30$ cycles of $1 \mathrm{~min}$ at $94^{\circ} \mathrm{C}, 1 \mathrm{~min}$ at $62^{\circ} \mathrm{C}$ and $1 \mathrm{~min}$ at $72^{\circ} \mathrm{C}$, with a final extension of $5 \mathrm{~min}$ at $72^{\circ} \mathrm{C}$.

PCR products were first electrophoresed in a $1.5 \%$ agarose gel to assure that a single band of 500-600 bp was present, then purified using the Real Clean Spin kit (REAL, Durviz S.L.U., Valencia, Spain), and finally bidirectionally sequenced on an ABI PRISM 3730xl automatic sequencer (Applied Biosystems, CA, USA) at the DNA sequencing services of Macrogen (Korea).

\section{Phylogenetic analyses}

Sequences and their individual secondary structures were obtained from the ITS2 Database [35-37]. Newly obtained ITS2 sequences were annotated according to Keller et al. [38], and their secondary structures predicted by homology modeling [39]. The phylogenetic analysis followed the procedure outlined in Schultz and Wolf [7] in accordance with Keller et al. [5]. The software used for the ITS2 sequence-structure analysis can be obtained from http://its2.bioapps.biozentrum.uniwuerzburg.de/?about. A global, multiple sequence-structure alignment was generated in 4SALE v1.5 $[40,41]$. The sequences and their individual secondary structures were synchronously aligned making use of an ITS2 sequence-structure specific scoring matrix [40], and the start and end of the alignment was manually adjusted. Based on primary and secondary structure information, phylogenetic relationships were reconstructed by ProfDistS, through the use of an ITS2 specific, general time reversible substitution model $[42,43]$. Bootstrap support [44] was estimated on 100 pseudo-replicates. The resulting tree was visualized with TreeView [45].

To study the species boundaries within Dunaliella we followed the "distinguishing species" instructions [6] 
based on compensatory base changes ( $\mathrm{CBCs}$ ) in the ITS2 secondary structure, and we used the CBCAnalyzer option implemented in 4SALE.

\section{Additional material}

Additional file 1: Compensatory Base Changes (CBC) analysis Compensatory Base Changes (CBCs) between different groups and species within the Dunaliella taxon (Excel file).

\section{Acknowledgements}

This research was supported by BANGEN-"Banco Genético de la Macaronesia", MAC/1/C070 (INTERREG-IIIB). We thank the Cabildo de Gran Canaria for allowing us to collaborate with the Departamento de Biodiversidad Molecular at the Jardín Botánico Canario "Viera y Clavijo"Unidad Asociada CSIC, and for continuous support to all its research lines. We would like to thank Matthias Wolf (University of Würzburg) for helping with the ITS2 sequence-structure analysis and to Frank Förster (University of Würzburg) for alignment adjustments.

\section{Author details}

'Departamento de Biotecnología. División de Investigación y Desarrollo Tecnológico. Instituto Tecnológico de Canarias (ITC). Pozo Izquierdo, 35119 Sta. Lucía, Canary Islands, Spain. ${ }^{2}$ Departamento de Biodiversidad Molecular y Banco de ADN, Jardín Botánico Canario "Viera y Clavijo"-Unidad Asociada CSIC, Apartado de correos 14 de Tafira Alta, 35017 Las Palmas de Gran Canaria, Canary Islands, Spain.

\section{Authors' contributions}

PA carried out the laboratory work, the phylogenetic analysis and wrote the manuscript. RJ-M and JC-C helped with the phylogenetic analysis and revised the manuscript. HM, AJ, LC and KF isolated the Dunaliella strains and revised the manuscript. HM conceived the study. All authors have read and approved the final manuscript.

\section{Competing interests}

The authors declare that they have no competing interests.

Received: 20 October 2011 Accepted: 30 January 2012

Published: 30 January 2012

\section{References}

1. Coleman AW, Suarez A, Goff L: Molecular delineation of species and syngens in volvocacean green algae (Chlorophyta). Journal of phycology 1994, 30:80-90.

2. Coleman AW, Mai JC: Ribosomal DNA ITS-1 and ITS-2 sequence comparisons as a tool for predicting genetic relatedness. Journal of Molecular Evolution 1997, 45:168-177.

3. Coleman AW: Pan-eukaryote ITS2 homologies revealed by RNA secondary structure. Nucleic Acids Research 2007, 35:3322-3329.

4. Coleman AW: ITS2 is a double-edged tool for eukaryote evolutionary comparisons. Trends in Genetics 2003, 19:370-375.

5. Keller A, Förster F, Müller T, Dandekar T, Schultz J, Wolf M: Including RNA Secondary Structures improves Accuracy and Robustness in Reconstruction of Phylogenetic Trees. Biology Direct 2010, 5:4.

6. Müller T, Philippi N, Dandekar T, Schultz J, Wolf M: Distinguishing species. RNA 2007, 13:1469-1472.

7. Schultz J, Wolf M: ITS2 Sequence-Structure Analysis in Phylogenetics: A How-to Manual for Molecular Systematics. Molecular Phylogenetics and Evolution 2009, 52:520-523.

8. Gomez Pl, Gonzalez MA: Genetic variation among seven strains of Dunaliella salina (Chlorophyta) with industrial potential, based on RAPD banding patterns and on nuclear ITS rDNA sequences. Aquaculture 2004, 233:149-162.
9. González MA, Gómez PI, Montoya R: Comparison of PCR-RFLP analysis of the ITS region with morphological criteria of various strains of Dunaliella. Journal of Applied Phycology 1999, 10:573-580.

10. González MA, Coleman AW, Gómez PI, Montoya R: Phylogenetic relashionship among strains of Dunaliella (Chlorophyceae) based on nuclear ITS rDNA sequences. Journal of Phycology 2001, 37:604-611.

11. González MA, Gómez PI, Polle JEW: Taxonomy and Phylogeny of the genus Dunaliella. In The Alga Dunaliella. Biodiversity, Physiology, Genomics and Biotechnology. Edited by: Ben-Amotz A, Polle JEW, Subba Rao DV. Science Publishers, Enfield, NH, USA; 2009:15-44.

12. Buchheim MA, Kirkwood AE, Buchheim JA, Verghese B, Henley WJ: Hypersaline soil supports a diverse community of Dunaliella (Chlorophyceae). Journal of Phycology 2010, 46:1038-1047.

13. Buchheim MA, Keller A, Koetschan C, Förster F, Merget B, Wolf M: Internal Transcribed Spacer 2 (nu ITS2 rRNA) sequence-structure phylogenetics: towards and automated reconstruction of the green algal tree of life. PLoS One 2011, 6:1-10.

14. Ben-Amotz A: Industrial production of microalgal cell-mass and secondary products - major industrial species. In Handbook of Microalgal Cultures, Biotechnology and Applied Phycology. Edited by: Richmond A. Blackwell, UK; 2004:273-280.

15. Ben-Amotz A, Avron M: The biotechnology of cultivating the halotolerant alga Dunaliella. Trends in Biotechnology 1990, 8:121-126.

16. Borowitzka MA, Borowitzka LJ: Dunaliella. In Microbial Biotechnology. Edited by: Borowitzka MA, Borowitzka L. Cambridge: Cambridge University Press; 1988:27-88.

17. Ben-Amotz A: Glycerol production in the alga Dunaliella. In Biochemical and Photosynthetic aspects of energy production. Edited by: san Pietro A. Academic Press, New York; 1980:191-208.

18. Massjuk NP: Morphology, Taxonomy, Ecology and Geographic Distribution of the Genus Dunaliella Teod. and Prospects for Its Potential Utilization. Naukova Dumka, Kiev [original in Russian] 1973, 242.

19. Cifuentes AS, González MA, Conejeros M, Dellarossa V, Parra OO: Growth and carotenogenesis in eight strains of Dunaliella salina Teodoresco from Chile. Journal of Applied Phycology 1992, 4:111-118.

20. Cifuentes AS, González M, Parra O: The effect of salinity on the growth and carotenogenesis in two Chilean strains of Dunaliella salina Teodoresco. Biological Research 1996, 29:227-236.

21. Cifuentes AS, González M, Parra O, Zúñiga M: Cultivo de cepas de Dunaliella salina (Teodoresco 1905) en diferentes medios bajo condiciones de laboratorio. Revista Chilena de Historia Natural 1996, 69:105-112.

22. Markovits A, Gianelli MP, Conejeros R, Erazo S: Strain selection for $\beta$ carotene production by Dunaliella. World Journal of Microbiology \& Biotechnology 1993, 9:534-537.

23. Gómez P, González M, Becerra J: Quantity and quality of $\beta$-carotene produced by two strains of Dunaliella salina (Teodoresco 1905) from the North of Chile. Boletín de la Sociedad Chilena Química 1999, 44:463-468.

24. Mendoza H, de la Jara A, Freijanes K, Carmona L, Ramos AA, de Sousa Duarte V, Serafim Varela JC: Characterization of Dunaliella salina strains by flow cytometry: a new approach to select carotenoid hyperproducing strains. Electronic Journal of Biotechnology 2008, 11(4), DOl: 10.2225/vol11issue4-fulltext-2 http://www.ejbiotechnology.info/content/vol11/issue4/full/ 2/2.pdf.

25. Gomez PI, Gonzalez MA: Genetic polymorphism in eight Chilean strains of the carotenogenic microalga Dunaliella salina Teodoresco (Chlorophyta). Biological Research 2001, 34:23-30.

26. Benson DA, Karsch-Mizrachi I, Lipman DJ, Ostell J, Sayers EW: GenBank. Nucleic Acids Research 2011, 39:D32-37.

27. Borowitzka MA, Siva CJ: The taxonomy of the genus Dunaliella (Chlorophyta, Dunaliellales) with emphasis on the marine and halophilic species. Journal of Applied Phycology 2007, 19:567-590.

28. Assunção P, Jaen-Molina R, Caujapé-Castells J, de la Jara A, Carmona L, Freijanes K, Mendoza H: Phylogenetic position Dunaliella acidophila (Chlorophyceae) based on ITS and rbcL sequences. Journal of Applied Phycology

29. Oren A: A hundred years of Dunaliella research: 1905-2005. Saline Systems 2005, 1(2)[http://www.salinesystems.org/content/1/1/2], doi: 10.1186/17461448-1-2. 
30. Coleman AW: The significance of a coincidence between evolutionary landmarks found in mating affinity and a DNA sequence. Protist 2000, 151:1-9.

31. Coleman AW: In there a molecular key to the level of "Biological species" in eukaryotes? A DNA guide. Molecular Phylogenetics and Evolution 2009, 50:197-203.

32. Polle JEW, Struwe L, Jin E: Identification and characterization of a new strain of the unicellular green alga Dunaliella salina (Teod.) from Korea. Journal of Microbiology and Biotechnology 2008, 18:821-827.

33. Richlen ML, Barber PH: A technique for the rapid extraction of microalgal DNA from single live and preserved cells. Molecular Ecology Notes 2005, 5:688-691.

34. Goff $\sqcup$, Moon DA, Coleman AW: Molecular delineation of species and species relationships in the red algal agarophytes Gracilariopsis and Gracilaria (Gracilariales). Journal of Phycology 1994, 30:521-537.

35. Schultz J, Müller T, Achtziger M, Seibel PN, Dandekar T, Wolf M: The internal transcribed spacer 2 database-a web server for (not only) low level phylogenetic analyses. Nucleic Acids Research 2006, , 34 Web Server: W704-707.

36. Selig C, Wolf M, Müller T, Dandekar T, Schultz J: The ITS2 Database II: homology modeling RNA structure for molecular systematics. Nucleic Acids Research 2008, 36:D377-380.

37. Koetschan C, Förster F, Keller A, Schleicher T, Ruderisch B, Schwarz R, Müller T, Wolf M, Schultz J: The ITS2 Database III - sequences and structures for phylogeny. Nucleic Acids Research 2010, 38:275-279.

38. Keller A, Schleicher T, Schultz J, Müller T, Dandekar T, Wolf M: 5.8S-28S rRNA interaction and HMM-based ITS2 annotation. Gene 2009, 430:50-57.

39. Wolf M, Achtziger M, Schultz J, Dandekar T, Müller T: Homology modeling revealed more than 20,000 rRNA internal transcribed spacer 2 (ITS2) secondary structures. RNA 2005, 11:1616-1623.

40. Seibel PN, Müller T, Dandekar T, Schultz J, Wolf M: 4SALE - A tool for synchronous RNA sequence and secondary structure alignment and editing. BMC Bioinformatics 2006, 7:498.

41. Seibel PN, Müller T, Dandekar T, Wolf M: Synchronous visual analysis and editing of RNA sequence and secondary structure alignments using 4SALE. BMC Research Notes 2008, 1:91.

42. Friedrich J, Dandekar T, Wolf M, Müller T: ProfDist: A tool for the construction of large phylogenetic trees based on profile distances. Bioinformatics 2005, 21:2108-2109.

43. Wolf M, Ruderisch B, Dandekar T, Müller T: ProfdistS: (Profile-) Distance based phylogeny on sequence-structure alignments. Bioinformatics 2008 24:2401-2402.

44. Felsenstein J: Confidence limits on phylogenies: An approach using the bootstrap. Evolution 1985, 39:783-791.

45. Page RDM: TreeView: an application to display phylogenetic trees on personal computers. Computer Applications in the Biosciences 1996, 12:357-358.

doi:10.1186/2046-9063-8-2

Cite this article as: Assunção et al:: Molecular taxonomy of Dunaliella (Chlorophyceae), with a special focus on D. salina: ITS2 sequences revisited with an extensive geographical sampling. Aquatic Biosystems 2012 8:2

\section{Submit your next manuscript to BioMed Central and take full advantage of:}

- Convenient online submission

- Thorough peer review

- No space constraints or color figure charges

- Immediate publication on acceptance

- Inclusion in PubMed, CAS, Scopus and Google Scholar

- Research which is freely available for redistribution 